\title{
A CLASS OF GENERALIZED UNIFORM ASYMPTOTIC EXPANSIONS
}

\author{
WILSON C. OBI \\ Department of Mathematics \\ Stevens Institute of Technology \\ Hoboken, New Jersey 07030
}

(Received May 2, 1977 and in revised form September 19, 1977)

ABSTRACT. In [1] E. L. Reiss obtained a multivariable expansion of the problem $L[y] \equiv y^{\prime \prime}+2 \varepsilon y^{\prime}+y=0, t>0 ; y(0 ; \varepsilon)=0, y^{\prime}(0, \varepsilon)=1$ by means of a technique which does not employ the traditional method of using a secularity condition in obtaining higher order approximations. In this paper Reiss' technique is formalized and new results are established.

0. INTRODUCTION.

$$
\begin{aligned}
& \text { Consider the problem } \\
& L[y]=y^{\prime \prime}+2 \varepsilon y^{\prime}+y=0, \quad\left({ }^{\prime}=\frac{d}{d t}\right), t>0 ; \\
& y(0 ; \varepsilon)=\gamma, y^{\prime}(0, \varepsilon)=\delta .
\end{aligned}
$$

where $\gamma$ and $\delta$ are constants independent of $\varepsilon$. This problem will be referred to as (P1). We seek a formal expansion of the form 


$$
y=\sum_{J=0}^{M} \varepsilon^{J} y^{J}\left(t_{0}, \ldots, t_{N}\right)+R^{M}(t ; \varepsilon)
$$

where the time scales are defined by

$$
t_{j}=\varepsilon^{j} t, j=0,1, \ldots, N
$$

Our task is to determine the coefficients $y^{J}$ so that $(0.3)$ constitutes a "generalized uniform asymptotic expansion" in an interval of $t$. Definitions to be given later will make clear the nature of these expansions.

When (0.3) and (0.4) are substituted into (0.1) we get an expression of the form

$$
L[y]=\sum_{\ell=0}^{M+2 N} \varepsilon^{\ell}\left[\sum_{\substack{J+k=\ell \\ 0 \leq J \leq M}} z_{k}^{J}\right]+L\left[R^{M}\right]=0
$$

where

$$
\begin{aligned}
& \mathrm{z}_{\mathrm{k}}^{\mathrm{J}}=0 \text { if } \mathrm{k}<0 \\
& z_{0}^{J}=y_{t_{0} t_{0}}^{J}+y^{J} \\
& z_{k}^{J}=\sum_{\substack{i+j=k \\
0 \leq i, j \leq N}} y_{t_{j} t_{i}}^{J}+2 y_{t_{k-1}}^{J}, 1 \leq k \leq N+1 \\
& z_{k}^{J}=\sum_{\substack{i+j=k \\
0 \leq i, j \leq N}} y_{t_{j} t_{i}}^{J} \quad, N+2 \leq k \leq 2 N \\
& \mathrm{z}_{\mathrm{k}}^{\mathrm{J}}=0 \quad, \mathrm{k}>2 \mathrm{~N}
\end{aligned}
$$

To determine the $y^{J} s$ we set the coefficients of $\varepsilon^{\ell}$ to zero for $\ell=0, \ldots, M$ while $M$ successively assumes the values $0,1,2 \ldots$. The solutions of the resulting equations involve arbitrary functions of $\mathrm{N}$ variables which must be found by imposing additional conditions. These conditions are supplied by further setting the coefficients of $\varepsilon^{M+1}, \ldots, \varepsilon^{M+N}$ to zero. Finally, to make the coefficients determinate $(0.3)$ is substituted into $(0.2)$ and the initial 
values of the $y^{J}$ s are chosen so that $(0.2)$ is satisfied as "closely" as possible. Specifically, when $(0.3)$ is substituted into $(0.2)$ we get

$$
\gamma=\sum_{J=0}^{M} \varepsilon^{J} y^{J}(\overrightarrow{0})+R^{M}(0)
$$

and

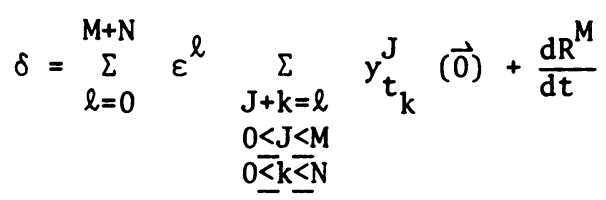

where $(\overrightarrow{0})$ denotes the vector $(0,0, \ldots, 0)$ corresponding to $t_{0}=t_{1}=\ldots=t_{N}$ $=0$. Equating the coefficients of like powers of $\varepsilon$ in the above as far as possible we obtain

$$
\begin{aligned}
& y^{\circ}(\overrightarrow{0})=\gamma, y_{t_{0}}^{o}(\overrightarrow{0})=\delta \\
& y^{J}(\overrightarrow{0})=0 ; \sum_{\substack{\mathrm{J}+\mathrm{k}=\ell \\
0<\mathrm{J}<\mathrm{M} \\
0 \leq k \leq \mathrm{N}}}^{\sum_{\mathrm{J}}} y_{t_{k}}^{\mathrm{J}}(\overrightarrow{0})=0 ; \mathrm{J}, \ell=1, \ldots, \mathrm{M}
\end{aligned}
$$

At the end of the formal process we are left with the following problem for the error term:

$$
\begin{aligned}
& L\left[R^{M}\right]=r^{M}(t ; \varepsilon)=-\sum_{\ell=M+N+1}^{M+2 N}\left\{\begin{array}{ccc}
\varepsilon^{\ell} & \sum_{\substack{J+k=\ell \\
0 \leq J \leq M}} z_{k}^{J}
\end{array}\right) \\
& \mathrm{R}^{\mathrm{M}}(0)=0, \frac{\mathrm{dR}}{\mathrm{dt}}(0)=\mathrm{Y}^{\mathrm{M}}=-\sum_{\ell=\mathrm{M}+1}^{\mathrm{M}+\mathrm{N}} \varepsilon^{\ell}\left(\sum_{\substack{\mathrm{J}+\mathrm{k}=\ell \\
0<\mathrm{J}<\mathrm{M} \\
0 \leq \mathrm{J}<\mathrm{N}}}^{\sum_{-\underline{k}}} \mathrm{y}_{\mathrm{t}_{\mathrm{k}}}^{\mathrm{J}}(\overrightarrow{0})\right)
\end{aligned}
$$

In this analysis we delineate explicitly the form of the coefficients $y^{J}$ and establish the uniform validity of the formal expansions over the infinite interval $[0, \infty)$. Estimates of the magnitude of the error are made from (0.9) and $(0.10)$. 
1. GENERAL DEPENDENCE OF COEFFICIENTS ON TIME SCALES

By setting the coefficients of $\varepsilon^{\ell}$ to zero in $(0.5)$ for $\ell=0, \ldots, M$, $\ldots, M+N$ as $M$ ranges through the values $0,1,2, \ldots$ it can be shown that for each $J$ we obtain

$$
\begin{aligned}
& \mathrm{z}_{\mathrm{k}}^{\mathrm{J}}=0, \mathrm{k}=0, \ldots, \mathrm{N}-1 \\
& \mathrm{z}_{\mathrm{N}}^{\mathrm{J}}=-\left\{\mathrm{z}_{\mathrm{J}+\mathrm{N}}^{0}+\ldots+\mathrm{z}_{\mathrm{N}+1}^{\mathrm{J}-1}\right\}
\end{aligned}
$$

The $(N+1)$ equations in (1.1) give the dependence of $y^{J}$ on the time scales $t_{0}$, $t_{1}, \ldots, t_{N}$, respectively. It is worth noting that dependence on $t_{0}, \ldots, t_{N-1}$ is the same for each $J$. We will now solve these equations to get the coefficients $y^{J}$. If we assume that $N$ is infinite, then we can explore the dependence of $y^{J}$ on $t_{0}, t_{1}, t_{2}, \ldots$ by solving $z_{0}^{J}=z_{1}^{J}=z_{2}^{J}=\ldots=0$. From (0.6) we obtain the first four equations:

$$
\begin{aligned}
& y_{t_{0} t_{0}}^{J}+y^{J}=0 \\
& y_{t_{0} t_{1}}^{J}+y_{t_{0}}^{J}=0 \\
& 2 y_{t_{0} t_{2}}^{J}+y_{t_{1} t_{1}}^{J}+2 y_{t_{1}}^{J}=0 \\
& 2 y_{t_{0} t_{3}}^{J}+2 y_{t_{1} t_{2}}^{J}+2 y_{t_{2}}^{J}=0
\end{aligned}
$$

The equations can be solved one after another. From (1.2) we get

$$
y^{J}=A^{1}\left(t_{1}, t_{2}, \ldots\right) e^{i t_{0}}+B^{1}\left(t_{1}, t_{2}, \ldots\right) e^{-i t_{0}}
$$

Now substitute (1.6) into (1.3) and require that (1.3) be satisfied identically. We obtain

$$
A^{1}=A^{2}\left(t_{2}, t_{3}, \ldots\right) e^{-t_{1}} ; B^{1}=B^{2}\left(t_{2}, t_{3}, \ldots\right) e^{-t_{1}} .
$$


Hence

$$
y^{J}=e^{-t_{1}}\left[A^{2}\left(t_{2}, \ldots\right) e^{i t_{0}}+B^{2}\left(t_{2}, \ldots\right) e^{-i t_{0}}\right]
$$

Similarly, we substitute (1.7) into (1.4) and so on. Proceeding in this manner we find that $y^{J}$ is sinusoidal in $t_{2}$ but is independent of $t_{3}$ (from (1.5)). If we carry the process still further we find that $y^{J}$ is sinusoidal in $t_{4}$ but is independent of $t_{5}$.

By induction we establish

$$
\begin{aligned}
& y^{J}=e^{-t_{1}}\left[A\left(t_{2(j+1)} \ldots\right) e^{i\left(t_{0}+\ldots+\alpha_{2 j} t_{2 j}\right)}\right. \\
& \left.+B\left(t_{2(j+1)}, \ldots\right) e^{-i\left(t_{0}+\ldots+\alpha_{2 j} t_{2 j}\right)}\right], j=1,2, \ldots \\
& \alpha_{0}=1, \alpha_{2}=-1 / 2, \\
& \alpha_{2 j}=-\frac{1}{2}\left(\alpha_{2} \alpha_{2(j-1)}+\ldots+\alpha_{2(j-1)} \alpha_{2}\right) \text {, if } j \geq 2
\end{aligned}
$$

and

We have therefore shown, by directly solving the governing differential equations, that when $\mathrm{N}$ is infinite the coefficients $\mathrm{y}^{\mathrm{J}}$ are sinusoidal in the even time scales $t_{0}, t_{2}, \ldots$ and independent of the odd scales $t_{3}, t_{5}, \ldots$. Dependence on $t_{1}$ is exponential as shown in (1.8).

A link between the form (1.8) and the exact solution of (P1) can be established. The exact solution is

$$
y=\gamma e^{-\varepsilon t} \cos \left(\sqrt{1-\varepsilon^{2} t}\right)+\frac{(\delta+\gamma \varepsilon)}{1-\varepsilon^{2}} e^{-\varepsilon t} \sin \left(\sqrt{1-\varepsilon^{2} t}\right)
$$

The desired link consists in showing from (1.9) and mathematical induction that $\alpha_{2 j}$ is the coefficient of $\varepsilon^{2 j}$ in the binominal expansion of $\left(1-\varepsilon^{2}\right)^{1 / 2}$. 
That is, we can write $\alpha_{2 j}$ alternatively as follows

$$
\alpha_{2 j}=(-1)^{j} \quad\left(\begin{array}{c}
1 / 2 \\
j
\end{array}\right)
$$

Now let us consider the practical case in which $\mathrm{N}$ is finite. As can be easily seen from (1.1), dependence on the time scales $t_{0}, \ldots, t_{N^{-1}}$ will be exactly as in the case where $\mathrm{N}$ is infinite. In order to be able to write expressions in a compact form, we will modify the definition for $\alpha_{2 j}$ when $2 \mathrm{j}>\mathrm{N}$. Let

$$
n=\left\{\begin{array}{l}
N / 2 \text { if } N \text { is even } \\
(N-1) / 2 \text { if } N \text { is odd }
\end{array}\right.
$$

Then

$$
\begin{aligned}
& \alpha_{2 j}=0 \text { if } j<0 \\
& \alpha_{0}=1, \alpha_{2}=-1 / 2 \\
& \alpha_{2 j}=-\frac{1}{2}\left(\alpha_{2} \alpha_{2(j-1)}+\ldots+\alpha_{2(j-1)} \alpha_{2}\right)=(-1)^{j}\left[\begin{array}{l}
\frac{1}{2} \\
j
\end{array}\right), 2 \leq j \leq n \\
& \alpha_{2 n+2 r}=-\frac{1}{2}\left(\alpha_{2 r} \alpha_{2 n}+\ldots+\alpha_{2 n} \alpha_{2 r}\right) \quad, 1 \leq r \leq n \\
& \alpha_{2 n+2 n}=-\frac{1}{2}\left(\alpha_{2 n}\right)^{2}, \quad, \quad r>n \\
& \alpha_{2 n+2 r}=0
\end{aligned}
$$

An immediate consequence of the results already established is that

$$
y^{J}=A^{J}\left(t_{1}\right) e^{i t_{0}}+B^{J}\left(t_{1}\right) e^{-i t_{0}}, N=1
$$


GENERALIZED UNIFORM ASYMPTOTIC EXPANSIONS

$$
\begin{aligned}
& y^{J}=e^{-t} 1\left[A^{J}\left(t_{N}\right) e^{i\left(t_{0}+\ldots+\alpha_{2(n-1)} t\right.} 2(n-1)\right) \\
& \left.+B^{J}\left(t_{N}\right) e^{-i\left(t_{0}+\ldots+\alpha_{2(n-1)} t_{2(n-1)}\right)}\right] N(\geq 2) \text { even } \\
& y^{J}=e^{-t_{1}}\left[A^{J}\left(t_{N}\right) e^{i\left(t_{0}+\ldots+\alpha_{2 n} t_{2 n}\right)}\right. \\
& \left.+B^{J}\left(t_{N}\right) e^{-i\left(t_{0}+\ldots+\alpha_{2 n} t_{2 n}\right)}\right] N(>1) \text { odd }
\end{aligned}
$$

In (1.15) and (1.16) $\mathrm{n}$ and. the frequencies $\alpha_{2 j}$ are given by (1.12) and (1.13), respectively.

After a lengthy but straightforward algebra it can be established that

$$
\begin{aligned}
y^{J}\left(t_{0}, \ldots, t_{N}\right)= & e^{-t_{1}}\left[P_{q}\left(t_{N}\right) e^{i\left(t_{0}+\ldots+\alpha_{2 n} t_{2 n}\right)}\right. \\
& \left.+\bar{P}_{q}\left(t_{N}\right) e^{-i\left(t_{0}+\ldots+\alpha_{2 n} t_{2 n}\right)}\right]
\end{aligned}
$$

where $P_{q}$ are complex valued polynomials in $t_{N}$, the last time scale in the set

$$
t_{j}=\varepsilon^{j} t, \quad j=0, \ldots, N
$$

and $n$ and the $\alpha_{2 i}$ are given by (1.12) and (1.13). The degrees of the polynomials depend on both $\mathrm{N}$ and $\mathrm{J}$ as follows

$$
q= \begin{cases}J / 2, & \text { if } J \text { is even and } N \text { is even } \\ (J-1) / 2, & \text { if } J \text { is odd and } N \text { is even } \\ J & , \text { if } N \text { is odd }\end{cases}
$$

The following form of $\mathrm{y}^{\mathrm{J}}$ is sometimes convenient to use:

$$
\begin{aligned}
y^{J}\left(t_{0}, \ldots, t_{N}\right) & =e^{-t} 1\left[2 \operatorname{Re}\left\{P_{q}\left(t_{N}\right)\right\} \cos \left(t_{0}+\ldots+\alpha_{2 n} t_{2 n}\right)\right. \\
& \left.-21 m\left\{P_{q}\left(t_{N}\right)\right\} \sin \left(t_{0}+\ldots+\alpha_{2 n} t_{2 n}\right)\right]
\end{aligned}
$$




\section{VALIDITY OF THE EXPANSIONS.}

We seek a "generalized uniform asymptotic expansion" (see [1] and [3]) in an interval $D$, which is assumed to be generally finite and possibly infinite. More specifically, we use the following definition

DEFINITION 1

Let $\left\{S_{j}(t ; \varepsilon)\right\}$ be a sequence of functions defined for $t \in D$ and $0 \leq \varepsilon$ $\leq \varepsilon_{M^{\circ}}$ for some $\varepsilon_{0}>0$, and let $\left\{f_{j}(\varepsilon)\right\}, \varepsilon \rightarrow 0$, be an asymptotic sequence. Then $\sum_{j=0} S_{j}(t ; \varepsilon)$ is a generalized Uniform asymptotic expansion of $y(t ; \varepsilon)$ in $D$ to $(M+1)$ terms if the remainders satisfy

$$
R^{k}(t ; \varepsilon)=y(t ; \varepsilon)-\sum_{j=0}^{k} S_{j}(t ; \varepsilon)=0\left(f_{k+1}\right)
$$

for each $k \in\{0,1, \ldots, M\}$.

In our analysis the element of the sequence, $S_{j}(t ; \varepsilon)$ will be in the form $\varepsilon^{J} y^{J}\left(t_{0}, \ldots, t_{N}\right)$ and $f_{k}=\varepsilon^{k}$. We wish to show that

$$
\sum_{J=0}^{M} \varepsilon^{J} y^{J}\left(t_{0}, \ldots, t_{N}\right)
$$

is a uniform asymptotic expansion of the solution of (P1) in the sense of (2.1) where the interval $D$ is infinite. To do this we need estimates of the error terms $R^{M}(t ; \varepsilon)$.

Since $L$ is a differential operator with constant coefficients, an integral representation of the solution of (0.9) can be obtained by means of the appropriate Green's function. With the integral representation estimates can be made as follows:

$$
\left|R^{M}(t ; \varepsilon)\right| \leq \alpha\left|Y^{M}\right|+(B / \varepsilon) \| r^{M}(t ; \varepsilon)||, t \geq 0
$$


where

$$
\|\cdot\|=\max _{t \geq 0}|\cdot|
$$

and $\alpha$ and $\beta$ are positive constants.

One of the goals of our analysis is to determine the link between $\left|R^{M}\right|$ and the initial conditions of $(P 1)$. This is achieved by expressing $Y^{M}$ in terms of those initial conditions. The following lemma gives the required expressions. Lemma .

The quantity $\frac{d R^{M}}{d t}(0)=Y^{M}$ can be written in the following terms

$$
\begin{aligned}
& \frac{d R^{M}}{d t}(0)=Y_{e}^{M}=\gamma \sum_{0 \leq s \leq[(N-1) / 2]}^{\sum_{2 s+1}} \varepsilon^{M+(2 s+1)} \\
&+\delta \sum_{1 \leq r \leq[N / 2]} \Pi_{2 r} \varepsilon^{M+2 r}, \text { if } M \text { is even } \\
& \frac{d R^{M}}{d t}(0)=Y_{0}^{M}=\delta \sum_{0 \leq s \leq[(N-1) / 2]} \Lambda_{2 s+1} \varepsilon^{M+(2 s+1)} \\
&+\gamma \sum_{1 \leq r \leq[N / 2]} \Lambda_{2 r} \varepsilon^{M+2 r}, \text { if } M \text { is odd }
\end{aligned}
$$

The constants $\Pi_{j}$ and $\Lambda_{j}$ depend on the quantities $\alpha_{2 i} \cdot[x]$ is defined as the greatest integer less than or equal to $x$.

The proof of the lemma is lengthy but straightforward and is omitted for brevity.

Before considering the main theorem, let us introduce an additional notation which will enable us to state the theorem in a compact form. Define the function $z_{\ell}(t ; \varepsilon)$ as follows: 


$$
\mathrm{z}_{\ell}(\mathrm{t} ; \varepsilon)=-\sum_{\substack{\mathrm{J}+\mathrm{k}=\ell \\ 0 \leq \mathrm{J} \leq \mathrm{M}}} \mathrm{z}_{\mathrm{k}}^{\mathrm{J}}
$$

Using (2.6) we can rewrite equation (0.9) in the form

$$
L\left[R^{M}\right]=r^{M}(t ; \varepsilon)=\sum_{\ell=M+N+1}^{M+2 N} \varepsilon^{\ell} z_{\ell}(t ; \varepsilon)
$$

Now let us state the theorem.

Theorem

If the time scales are given by $(0.4)$, then for $N \geq 1(0.3)$ is a $(M+1)$ - term - $(\mathrm{N}+1)$ - time generalized uniform asymptotic expansion of the solution of $(P 1)$ in the interval $[0, \infty)$. The error $R^{M}(t ; \varepsilon)$ is bounded in magnitude as follows:

$$
\begin{aligned}
\left|R^{M}(t ; \varepsilon)\right| & \leq A_{1}\left|Y_{e}^{M}\right|+\left(B_{1} / \varepsilon\right)\left\|r^{M}(t ; \varepsilon)\right\|, \text { if } M \text { is even } \\
& \leq A_{2}\left|Y_{0}^{M}\right|+\left(B_{2} / \varepsilon\right)\left\|r^{M}(t ; \varepsilon)\right\| \text {, if } M \text { is odd }
\end{aligned}
$$

where $Y_{e}^{M}$ and $Y_{0}^{M}$ are given by (2.4) and (2.5), respectively;

$$
\left\|\mathrm{r}^{\mathrm{M}}(\mathrm{t} ; \varepsilon)\right\| \leq \sum_{\ell=M+N+1}^{\mathrm{M}+2 \mathrm{~N}} \varepsilon^{\ell}\left\|\mathrm{z}_{\ell}(t ; \varepsilon)\right\|
$$

and $A_{1}, A_{2}, B_{1}, B_{2}$ are positive constants.

Proof

From equations $(0.9),(2.2)-(2.5)$ we see that the theorem will be proved if we can show that

$$
\left\|z_{\ell}(t ; \varepsilon)\right\|=\left\|\sum_{\substack{\mathrm{J}+\mathrm{k}=\ell \\ 0 \leq \mathrm{J} \leq \mathrm{M}}} \mathrm{z}_{\mathrm{k}}^{\mathrm{J}}\right\|
$$

exists for all $t \geq 0$. From (0.6) we see that for each $\ell, z_{\ell}$ is a sum of terms like $y^{J}, y_{t_{k}}^{J}$ and $y_{t_{j} t_{i}}^{J}$; these terms are therefore of the form 


$$
t_{N}^{q} e^{-t_{1}}\left\{\begin{array}{l}
\sin \left(t_{0}+\ldots+\alpha_{2 n} t_{2 n}\right) \\
\cos \left(t_{0}+\ldots+\alpha_{2 n} t_{2 n}\right)
\end{array}\right\}
$$

where $q \leq M$. With $t_{1}=\varepsilon t$ and $t_{N}=\varepsilon^{N} t$ it can be shown that the quantities in (2.10) are bounded by $\left(\varepsilon^{N-q}\right) q^{q} e^{-q}$. for $t \geq 0$. Therefore $\left\|z_{\ell}(t ; \varepsilon)\right\|$ exists. (2.7) follows from the lemma and equation (2.2) while (2.8) follows from (0.9b) and the triangle inequality. Thus the theorem is proved. Q.E.D.

The following corollaries are easily established.

Corollary 2.

If $N=1$, then $R^{M}=0\left(\varepsilon^{M+1}\right)$ for all $M$.

Corollary 3.

Let $N$ be greater than 1 and $\gamma=0$. Then,
(I) $R^{M}=0\left(\varepsilon^{M+1}\right)$ if $M$ is odd
(II) $R^{M}=0\left(\varepsilon^{M+2}\right)$ if $M$ is even
(III) $y^{\mathrm{J}}=0$ when $\mathrm{J}$ is odd and $\mathrm{N}$ is even.

Corollary 4.

Let $\mathrm{N}$ be greater than 1 and $\delta=0$. Then

$$
\begin{aligned}
& \text { (IV) } R^{M}=0\left(\varepsilon^{M+2}\right) \text { if } M \text { is odd } \\
& \text { (V) } R^{M}=0\left(\varepsilon^{M+1}\right) \text { if } M \text { is even }
\end{aligned}
$$

\section{GENERAL RESULTS}

The first set of results to be presented in this section concerns the role of the number of time scales in the expansions. We give and clarify the following results originally stated by Reiss [1]:

There are a minimum number of time scales, namely two, that are required to obtain a uniform asymptotic expansion. 
For a fixed number of terms in the expansion three or more time scales give uniform expansions with the smallest estimate of the remainder. In particular, three time scales will always give uniform expansions with the smallest estimate of the remainder.

From (RII) it is seen that there may be no justification in using $N>2$ (i.e. more than three time scales). However, expansions using $\mathrm{N}>2$ may be desirable for certain purposes as is the case when more accurate frequencies are desired. Recall the error bound given in (2.7) and (2.8). One of the terms in this bound consists of a sum of terms of the form

$$
\left(\varepsilon^{N+l-q}\right) q^{q} e^{-q}, 0 \leq q \leq M, M+N+1 \leq \ell \leq M+2 N
$$

Thus for fixed $M$ this term can be decreased indefinitely by simply increasing the number of time scales. Only the term depending on the initial conditions cannot be so diminished. In a given initial value problem ( $M$ fixed) is it legitimate to ask if we can select the least number of time scales which give the smallest estimate of the remainder? Levine and Lubot [4] and obi [2] have explored this question in some detail. The answer is in the affirmative. In many cases three is the least number, but with certain initial conditions (which depend upon $\varepsilon$ ) one can indeed increase the accuracy indefinitely by merely increasing the number of time scales (even for $M=0$ ). We now turn to the role of the odd scales $\varepsilon^{3} t, \varepsilon^{5} t, \ldots$ in the multitime expansions. Reiss had reasoned that since these scales do not appear in the expansions of the exact solution the multi-time method need be applied using only the scales

$$
t_{0}=t, t_{1}=\varepsilon t, \ldots, t_{k}=\varepsilon^{2(k-1)} t, \quad k=2,3, \ldots N
$$

The present analysis clearly shows that expansions involving the odd scales 
exist. We reproduce some of them:

$$
\begin{aligned}
y & =y^{o}\left(t_{0}, t_{1}, t_{2}, t_{3}\right)+\varepsilon y^{1}\left(t_{0}, \ldots, t_{3}\right)+0\left(\varepsilon^{2}\right) \\
& =e^{-t_{1}} \sin \left(t_{0}-\frac{1}{2} t_{2}\right)+\varepsilon\left[-\frac{1}{8} t_{3} e^{-t_{1}} \cos \left(t_{0}-\frac{1}{2} t_{2}\right)\right]+0\left(\varepsilon^{2}\right) \\
y & =y^{o}\left(t_{0}, \ldots, t_{3}\right)+\varepsilon y^{1}\left(t_{0}, \ldots, t_{3}\right)+\varepsilon^{2} y^{2}\left(t_{0}, \ldots, t_{3}\right)+0\left(\varepsilon^{4}\right) \\
& =e^{-t_{1}} \sin \left(t_{0}-\frac{1}{2} t_{2}\right)+\varepsilon\left[-\frac{1}{8} t_{3} e^{-t_{1}} \cos \left(t_{0}-\frac{1}{2} t_{2}\right)\right] \\
& +\varepsilon^{2}\left[\frac{1}{2}\left(1-\frac{1}{64} t_{3}^{2}\right) e^{-t_{1}} \sin \left(t_{0}-\frac{1}{2} t_{2}\right)\right]+0\left(\varepsilon^{4}\right)
\end{aligned}
$$

In these expansions $\gamma=0, \delta=1$ and $t_{k}$ is given by $(0.4)$. Observe that $R^{0}=$ $O\left(\varepsilon^{2}\right)$ and $R^{1}=O\left(\varepsilon^{2}\right)$ so that Corollary 3 still holds. This will appear confusing at first until we further observe that in reality $y^{1}$ is "small". Since $t_{3} e^{-t}=\varepsilon^{3} t e^{-\varepsilon t}$ and $t e^{-\varepsilon t}$ is uniformly bounded we see that $\varepsilon y^{1}=0\left(\varepsilon^{4}\right)$. Hence this term does not improve the order of magnitude estimate of the error. The approach taken in this analysis has thus revealed a larger class of generalized uniform asymptotic expansions. The results of [1] can be recovered in one of two ways. The first is to work with the complete set $(0.4)$ but with $\mathrm{N}$ even. In this case dependence on the odd scales drops out in direct computations. Another way is to seek expansions of the form

$$
y=y^{o}\left(t_{0}, \ldots, t_{3}\right)+\varepsilon^{2} y^{2}\left(t_{0}, \ldots, t_{3}\right)+\bar{R}^{2}
$$

That is, we assume beforehand that the coefficient with the odd superscript vanishes. In this case, however, we obtain the same expansion as would result if we used only $t_{0}, t_{1}, t_{2}$. (This should be regarded as a coincidence since such results do not carry over when we consider equations with variable coefficients [2]). 
The role of the initial conditions in determining the approximations is very interesting. When $\mathrm{N}(\geq 2)$ is even the coefficients $\mathrm{y}^{\mathrm{J}}, \mathrm{J}$ an odd integer, are all proportional to $\gamma=y(0)$. These coefficients therefore vanish when $\gamma=$ 0 but not otherwise (constrast Corollaries 3 and 4). The analysis in [1] considered only the case $\gamma=0$ and the incomplete picture thus presented certainly gave rise to the false impression that the odd coefficients will vanish for other cases.

Another important set of observations concerning the influence of the number of time scales is fundamental to the understanding of multi-time expansions. This analysis shows clearly two kinds of dependence on individual time scales. The first kind is definitely a property of the differential equation involved. Thus if we assume that $N$ is infinite only $t_{0}, t_{1}$ and the even scales appear in our expansions. These scales are precisely those which will appear in the uniformly valid expansions of the exact solution. The second kind of dependence may be characterized as a truncation effect. This is a consequence of using only a finite number of time scales and manifests itself in the form of polynominals in the last scale, $t_{N}$. It is due to this effect that we are able to get a class of expansions involving the other odd scales. These expansions were not anticipated, and in fact, were discounted in [1].

\section{FINAL REMARKS}

The general results reported in this paper are common to a broad class of generalized uniform asymptotic expansions. The basic feature of the method reported in [1] is found to be the imposition of secularity conditions by picking approximations of a certain form. The most general form of such approximations have been dealt with in [5] and [6] where it has been shown that time 
scales may be derived from the governing differential equation.

\section{ACKNOWLEDGEMENT}

The author expresses his sincerest appreciation to Professor L. E. Levine for his encouragement and guidance. This work was done while the author was a recipient of the Robert Crooks Stanely Fellowship at Stevens Institute of Technology.

\section{REFERENCES}

[1] Reiss, E. L., "On Multivariable Asymptotic Expansions", SIAM Review, Vol. 13, No. 2, April 1971, pp. 189-196.

[2] Obi, W. C., "A Diminishing Error Approach to Multivariable Expansions of Second Order Linear Differential Equations", Doctoral Thesis, Stevens Institute of Technology, Hoboken, N.J., 1975

[3] Erdelyi, A., "Two-Variable Expansions for Singular Perturbations", J. Inst. Maths. Applic., 4, 1968, pp. 113-119.

[4] Levine, L. E. and Lubot, E. S., "Optimal Approximate Solutions and Time Scales", SIAM J. App. Math.. Vol. 29, No. 3, November 1975.

[5] Levine, L. E. and Obi, W. C., "Multivariable Expansion of Solutions of Linear Equations with Slowly Varying Coefficients", Bull. Amer. Math. Soc., Vo1. 82, No. 5, September 1976.

[6] Levine, L. E. and Obi, W. C., "Multivariable Expansions Using Time Scales Generated from Differential Equations", SIAM J. App. Math., Vol. 33, No. 4, December 1977.

KEY WORDS AND PHRASES. Asymptotic expansions, ordinary linear differential equations, uniform asymptotic expansions, and multivariable expansions. 


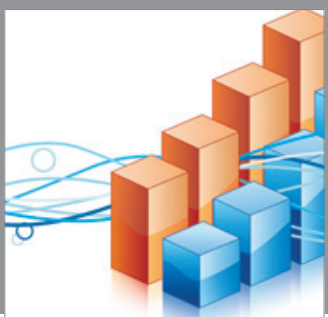

Advances in

Operations Research

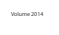

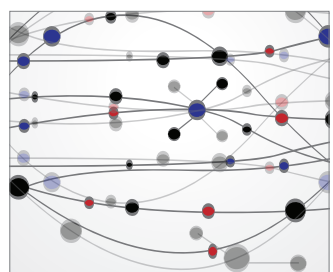

\section{The Scientific} World Journal
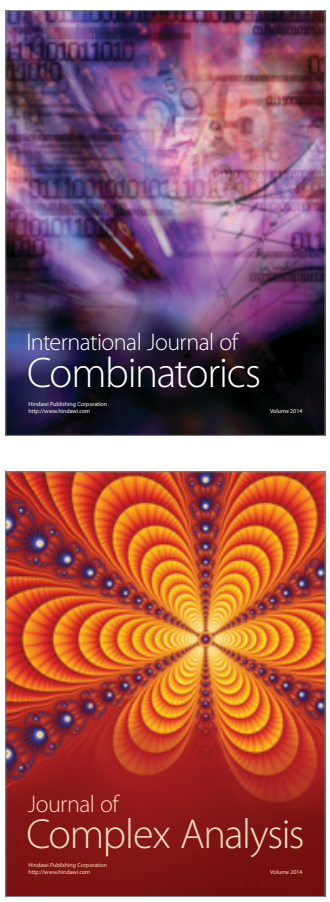

International Journal of

Mathematics and

Mathematical

Sciences
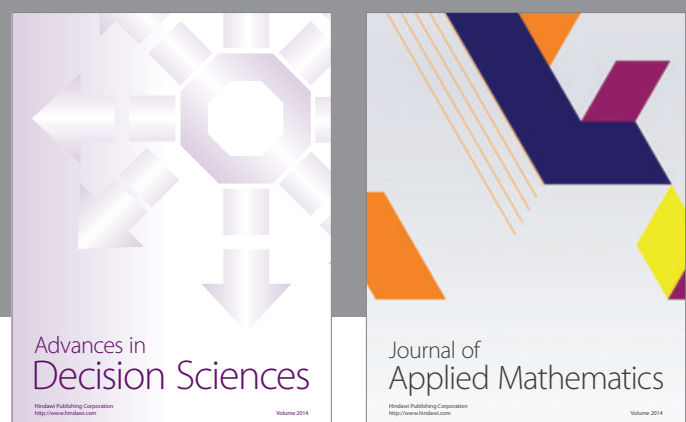

Journal of

Applied Mathematics
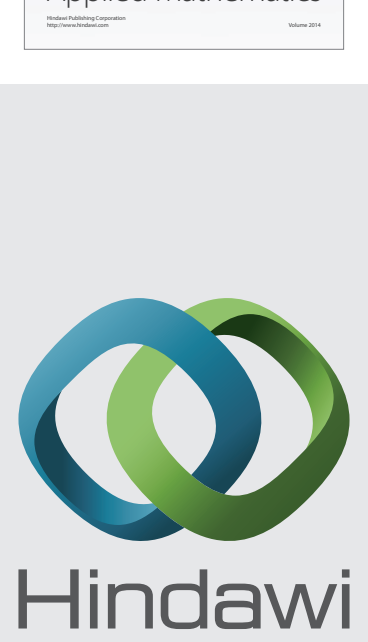

Submit your manuscripts at http://www.hindawi.com
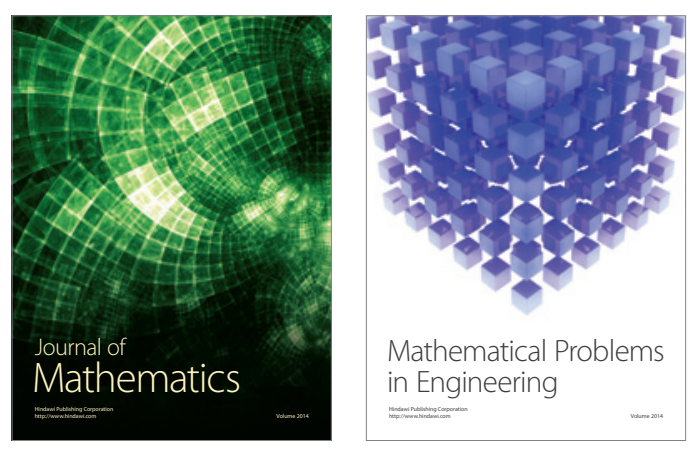

Mathematical Problems in Engineering
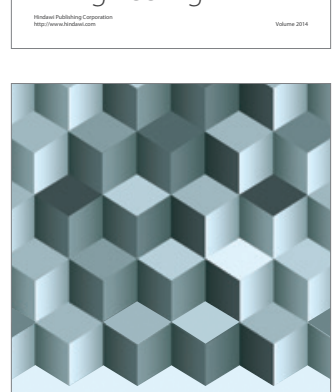

Journal of

Function Spaces
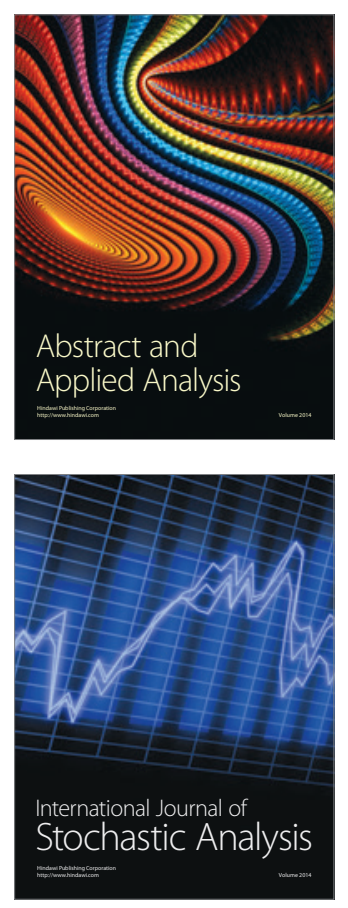

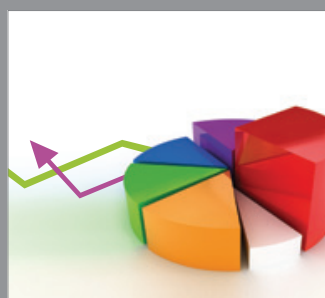

ournal of

Probability and Statistics

Promensencen
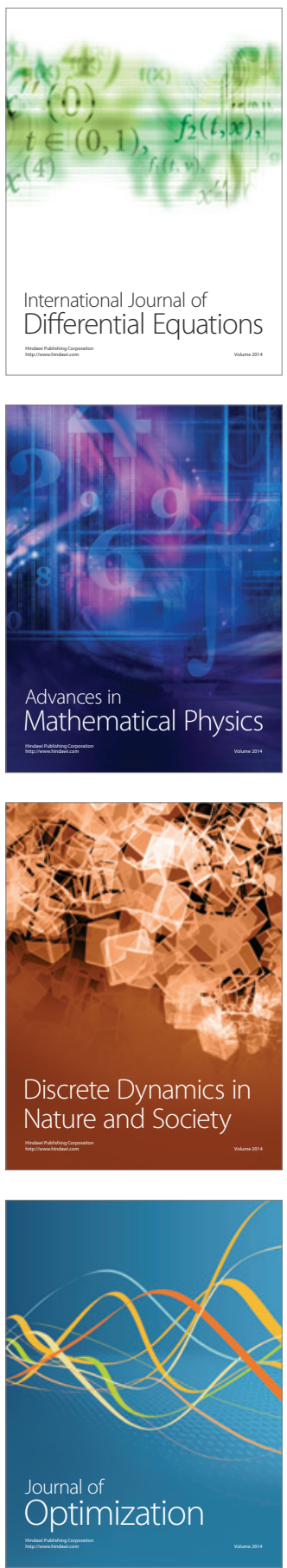